\title{
Optical Description of Mesostructured Organic-Inorganic Halide Perovskite Solar Cells
}

\author{
Miguel Anaya, ${ }^{\dagger}$ Gabriel Lozano, ${ }^{\dagger}$ Mauricio E. Calvo, ${ }^{\dagger}$ Wei Zhang, ${ }^{\ddagger}$ Michael B. Johnston, ${ }^{\ddagger}$ Henry J. Snaith, ${ }^{\ddagger}$ \\ and Hernán Míguez ${ }^{* \dagger}$ \\ ${ }^{\dagger}$ Instituto de Ciencia de Materiales de Sevilla, Consejo Superior de Investigaciones Científicas (CSIC), Universidad de Sevilla, Calle \\ Américo Vespucio 49, 41092 Sevilla, Spain \\ ${ }^{\ddagger}$ Department of Physics, University of Oxford, Clarendon Laboratory, Parks Road, Oxford X1 3PU, United Kingdom
}

\section{Supporting Information}

ABSTRACT: Herein we describe both theoretically and experimentally the optical response of solution-processed organic-inorganic halide perovskite solar cells based on mesostructured scaffolds. We develop a rigorous theoretical model using a method based on the propagation of waves in layered media, which allows visualizing the way in which light is spatially distributed across the device and serves to quantify the fraction of light absorbed by each medium comprising the cell. The discrimination between productive and parasitic absorption yields an accurate determination of the internal quantum efficiency. State-of-the-art devices integrating mesoporous scaffolds infiltrated with perovskite are manufactured and characterized to support the calculations. This combined experimental and theoretical analysis provides a rational understanding of the optical behavior of perovskite cells and can be beneficial for the judicious design of devices with improved performance. Notably, our model justifies the presence of a solid

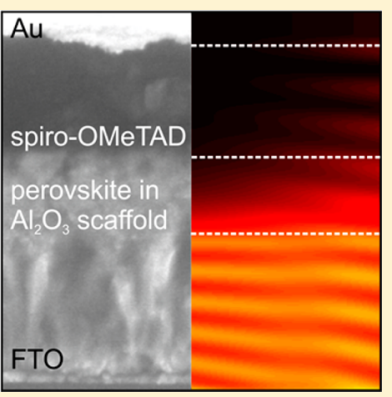
perovskite capping layer in all of the highest efficiency perovskite solar cells based on thinner mesoporous scaffolds.

$\mathrm{O}$ rganic-inorganic halide perovskite solar cells have driven a paradigm shift in photovoltaics (PV) since they are breaking with the sempiternal trade-off between power conversion efficiency and fabrication cost. ${ }^{1-5}$ Not long after their inception, this solar cell concept is already striding ahead in the emerging PV efficiency race, having reached in the last 2 years certified efficiency values that significantly surpass all of the ones attained for other cells based on solution-processed materials and rapidly approaching existing commercial thin-film technologies. ${ }^{6}$ This rapid progress has been based on improvements of fabrication methods, charge carrier materials, device architecture, morphology and crystallinity of the perovskite thin films, and the composition of the perovskite. $^{7-19}$ Another route to boost the efficiency of solar cell devices consists of the optimization of their optical design to maximize light harvesting and charge collection. ${ }^{20}$ In this regard, efforts have been made to optimize the electro-optical performance of both materials ${ }^{21-24}$ and devices. ${ }^{25}$ In this respect, only cells that make use of flat, evaporated, perovskite layers have been modeled and, to the best of our knowledge, a detailed description of the optical behavior of the complete mesostructured device is still missing, especially understanding the difference between the optical properties of the perovskite when forming a solid crystalline film, in comparison with when infiltrated into a mesoporous scaffold.

Herein we perform an in-depth experimental and theoretical analysis of the optical effects occurring in state-of-the-art solution-processed organic lead halide perovskite cells. We develop a rigorous theoretical model based on the transfer matrix formalism, ${ }^{26}$ which allows us to calculate the electric field intensity within the layered structure and thus to visualize the effect of each component of the cell on the spread of the radiation in the device. This permits us to estimate the amount of light that is harvested by each absorbing layer in the cell and consequently to distinguish between productive and parasitic absorption in the PV device. Such discrimination enables the accurate determination of the internal quantum efficiency, which otherwise would be underestimated. To check the suitability of the proposed theoretical model, we compare our results with the experimental reflectance, transmittance, and absorptance spectra of actual cells. Our work also provides a theoretical framework in which the optical response of devices integrating photonic enhancing components can be rationalized. We believe such integration may open the door to the realization of perovskite solar cells of improved efficiency due to the maximization of light absorption in extremely thin films.

Figure 1a shows a secondary electron scanning electron microscopy (SEM) image of a cross section of a perovskite solar cell, and Figure $1 \mathrm{~b}$ shows a schematic of such PV device. From the bottom side, where the light impinges, the cell is composed of a fluorine-doped tin oxide (FTO)-coated glass, covered by an n-type electron-collecting $\mathrm{TiO}_{2}$ compact layer that also acts as a blocking layer. $\mathrm{A}$ mesoporous $\mathrm{Al}_{2} \mathrm{O}_{3}$ layer, which serves as scaffold, is then deposited over the n-type layer, followed by the infiltration of the $\mathrm{CH}_{3} \mathrm{NH}_{3} \mathrm{PbI}_{3}$ perovskite material. Please note that solution-processed films typically

Received: November 5, 2014

Accepted: December 4, 2014

Published: December 4, 2014 
(a)

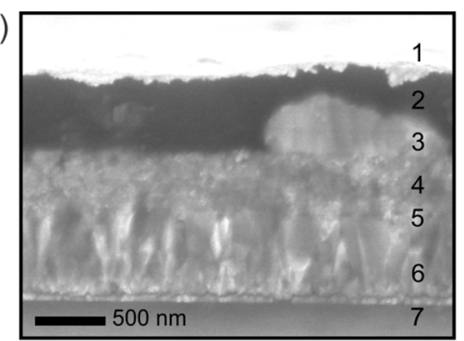

(b)

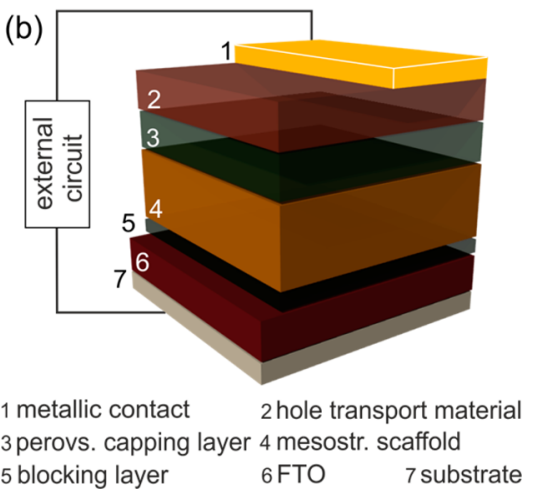

Figure 1. (a) Secondary electron scanning electron microscope image of a cross section of a representative solution-processed perovskite solar cell that integrates a ca. $350 \mathrm{~nm}$ thick mesoporous scaffold. It is composed of gold as metallic contact, spiro-OMeTAD as hole transport material, a mesostructured $\mathrm{Al}_{2} \mathrm{O}_{3}$ scaffold infiltrated by $\mathrm{CH}_{3} \mathrm{NH}_{3} \mathrm{PbI}_{3}$, and compact $\mathrm{TiO}_{2}$ as a blocking layer. The cell is deposited over a fluorinated tin oxide (FTO) coated glass substrate. (b) Schematic of the device.

show a perovskite capping layer, which can be nonuniform in nature, over the scaffold, as we show in Figure 1a. The holetansporting material, 2,2',7,7'-tetrakis-( $N, N$-di- $p$-methoxyphenylamine) $9,9^{\prime}$-spirobifluorene (spiro-OMeTAD), is then deposited over the perovskite to collect the holes at the gold cathode. We measure the PV performance of the solar cells under investigation. The average device of a batch of 22 cells fabricated in an identical way had a short-circuit photocurrent of $21.7 \pm 1.5 \mathrm{~mA} \mathrm{~cm}^{-2}$, an open-circuit voltage of $1.02 \pm 0.02$ $\mathrm{V}$, and a fill factor of $0.63 \pm 0.02$, yielding an efficiency of 13.8 $\pm 1.1 \%$ that exhibited a good stabilized power output. The complete PV characterization is provided in the Supporting Information (see Figures S1 and S2).

The perovskite cell is herein modeled through a layered structure, as depicted in Figure 1b. The incoming and outgoing media are considered to be air. In our model, the optical effects that arise from the presence of all layers comprising the PV device are accounted for. The optical constants of the different constituents of the stack, that is, glass, FTO, spiro-OMeTAD, and $\mathrm{Au}$, are taken from literature. ${ }^{27-29}$ The spectral dependence of the perovskite complex refractive index $(N=n+i \cdot k)$ is shown in Figure 2a. Values of the real $(n)$ and imaginary part $(k)$ are attained by the simultaneous fitting of the Fresnel equations to the reflectance and transmittance measured at normal incidence from thin layers of the perovskite absorber deposited over glass substrates. The attained wavelength-bywavelength $N$ is employed to calculate a real and an imaginary part that are Kramers-Kronig consistent. Details are provided in the Supporting Information. The optical constants displayed in Figure 2a are in good agreement with those reported for a similar material in refs 21,25 , and 30 . Similar spectral features are observed in both the real and the imaginary part. However,
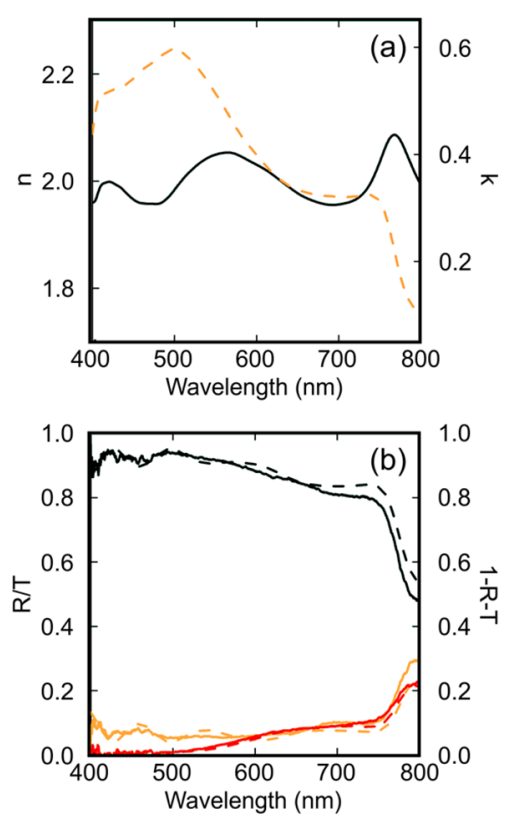

Figure 2. (a) Spectral dependence of the real (black solid line) and imaginary part (orange dashed line) of the refractive index of the $\mathrm{CH}_{3} \mathrm{NH}_{3} \mathrm{PbI}_{3}$ perovskite absorber. Values were obtained from fits of the Fresnel equations to reflectance $(R)$ and transmittance $(T)$ spectra measured at normal incidence from thin layers of perovskite. (b) $R$ (orange lines), $T$ (red lines), and total absorptance (1-R-T) spectra (black lines) calculated (dashed lines) and measured (solid lines) from a representative device comprising a $350 \mathrm{~nm}$ thick mesoporous $\mathrm{Al}_{2} \mathrm{O}_{3}$ scaffold infiltrated by perovskite.

although the values attained are quantitatively comparable for the extinction coefficient, a clear difference in the mean value of the real part, ranging between 2 and 2.5, is observed between the different reported data. (See Figure S4 in the Supporting Information.) We attribute this discrepancy to the different experimental routes, from wet chemistry to vapor deposition, followed to prepare the perovskite absorber.

We employ an effective medium approximation to account for: (i) the rough FTO surface coated by the thin compact layer, (ii) the perovskite-infiltrated mesostructured scaffold, and (iii) the nonuniform nature of the interface between the perovskite overlayer and the spiro-OMeTAD layer. To determine the effective optical constants of such media, we considered different equations, that is, volume-weighted average, Cuming and Maxwell-Garnett. Regarding (i) and (iii), in view of the cross sections observed in the SEM, considering an equal contribution to the refractive index of the transition middle layer from the materials at both sides of the interface seems reasonable. In the case of the mesostructured $\mathrm{Al}_{2} \mathrm{O}_{3}$ scaffold, a $54 \%$ porosity prior to perovskite infiltration was attained from the fitting of its experimental reflectance, assuming that the thickness and the permittivity of the alumina are known from the analysis of SEM images and the scientific literature, ${ }^{31}$ respectively. The very similar results attained from the different effective medium equations employed led us to consider the simplest approximation, that is, the volumeweighted average, to calculate the effective optical constants.

Our theoretical model serves to describe the optical response, that is, reflectance $(R)$, transmittance $(T)$, and absorptance $(A)$, of a representative cell shown as, respectively, orange, red, and black solid lines in Figure $2 \mathrm{~b}$. Measurements performed in an integrating sphere show that the $A$ of the 
device reaches values above $90 \%$ at $500 \mathrm{~nm}$, where the extinction coefficient is maximum, shows values above $80 \%$ from 400 to $700 \mathrm{~nm}$, and significantly diminishes at ca. $800 \mathrm{~nm}$, at the edge of the perovskite absorber electronic band gap. As in the experiments, the calculation considers an incident wave vector that impinges perpendicularly to the electrode and therefore to the stacking of dielectric layers, although the description of angular and polarization effects would also be possible if necessary. To obtain the $R$ and $T$ of the cell, we calculated the intensity of the electric field in the incident and outgoing medium, respectively, by solving the set of equations established by imposing the continuity of the electric and magnetic fields across each interface using a method based on the transfer matrix formalism. (See ref 32 for details.) Notice that the thick glass substrate can be considered in the model as a layer that does not give rise to interference effects because the coherence length of the incident light is much shorter than its thickness. ${ }^{33}$ As a result, the calculated spectra in Figure $2 \mathrm{~b}$ do not show the very short period fringes, which would arise from the presence of an ideal flat ca. $1 \mathrm{~mm}$ thick layer. The total absorptance is estimated as the fraction of light that was neither reflected nor transmitted as $1-R-T$. A linear least-squares method is employed to fit the experimental $R$ and $T$. We consider the thickness of the different layers comprising the multilayer stack as fitting parameters. The values extracted from the SEM are taken as initial values. Fair agreement between theory (dashed lines) and experiment (solid lines) is found as displayed in Figure $2 \mathrm{~b}$. The correspondence between spectral features in $R$ and $A$ can be clearly seen. As the wavelength increases, the extinction coefficient of the perovskite diminishes, and both $R$ and $T$ increase. Note that our model does not account for the effect of diffuse scattering. In fact, the diffuse reflectance may well explain the small discrepancy observed between measured and calculated $R$ and, consequently, between measured and calculated $A$ in the spectral range comprised between ca. 700 and $800 \mathrm{~nm}$, where the fraction of light diffusely reflected shows values between ca. 2 and $10 \%$. This results in an overestimation of the fraction of light absorbed by the device at longer wavelengths. Note that the air mass coefficient in this range greatly reduces and therefore the impact of the inaccuracy of our model in the overall response of the solar cell is not significant. The spectral dependence of the experimental total and diffuse reflectance is shown in Figure S5 of the Supporting Information.

We have also calculated the spatial distribution of the square magnitude of the electric field in the multilayer stack. Figure 3a shows the calculated spatial (vertical axis) and spectral (horizontal axis) distribution of the electric field intensity enhancement $\left(I / I_{0}\right)$, that is, near-field intensity normalized by the incident intensity, along a cross section of the PV device. Light impinges from the bottom. The glass-FTO interface is sited at $0 \mu \mathrm{m}$ in the vertical axis. The interference of all beams reflected and transmitted at each interface determines the way in which the radiation distributes over the solar cell for each wavelength. Different wavelengths propagate distinctly through the device. We can observe that the intensity at ca. $500 \mathrm{~nm}$ decreases very rapidly in the absorbing scaffold until it vanishes within the perovskite overlayer. In contrast, at $750 \mathrm{~nm}$, where the extinction coefficient of the perovskite absorber is reduced, the penetration of the optical field in the device is significantly larger. The incoming light reaches the last layers of the solar cell, which yields a nonzero transmittance; see Figure $2 \mathrm{~b}$. The calculation of the spatial distribution of the electric field
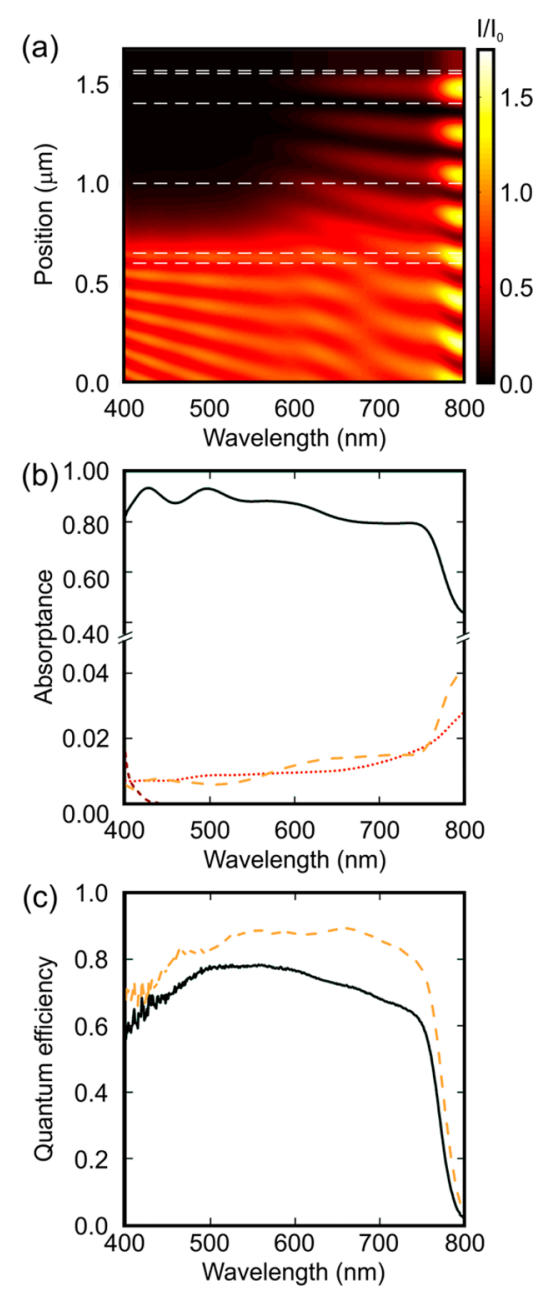

Figure 3. (a) Calculated spatial (vertical axis) and spectral (horizontal axis) distribution of the electric field intensity enhancement $\left(I / I_{0}\right)$, that is, near-field intensity normalized by the incident intensity, along a cross section of the perovskite solar cell. Calculations were carried out considering the multilayer parameters extracted from the fittings to reflectance and transmittance measurements. Horizontal dashed lines are guides to the eye to delimit the interfaces between media in the device. From top to bottom: air, $\mathrm{Au}$, spiro-OMeTAD, perovskite overlayer, perovskite-infiltrated $\mathrm{Al}_{2} \mathrm{O}_{3}$ scaffold, $\mathrm{TiO}_{2}$ compact layer, and FTO. (b) Spectral dependence of the fraction of light absorbed by the different absorbing materials comprising the device: FTO (red dotted line), spiro-OMeTAD (brown short dashed line), perovskite (black solid line), and Au (orange dashed line). (c) Experimental external quantum efficiency (black solid line) and internal quantum efficiency (orange dashed line) estimated from the calculation of the fraction of light absorbed by the perovskite material.

intensity inside the solar cell allows us to compute the fraction of light absorbed by each constituent of the PV device $\left(A_{\mathrm{j}}\right)$ according to

$$
A_{j}=2 \pi \int_{x_{j}} \frac{I\left(x_{j}, \lambda\right)}{I_{0}(\lambda)} \frac{2 n_{j} k_{j}}{\lambda} \mathrm{d} x_{j}
$$

where the subscript $j$ refers to each layer comprising the cell and $\lambda$ is the wavelength of the radiation. The integral extends over the volume occupied by each medium, which can be reduced to one dimension $\left(x_{\mathfrak{j}}\right)$ due to the symmetry of the system. Figure $3 \mathrm{~b}$ displays the fraction of light absorbed by the different absorbing materials that constitute the cell, that is, FTO (red dotted line), spiro-OMeTAD (brown short dashed 
line), perovskite (black solid line), and Au (orange dashed line). Thus, our model permits us to quantify the optical losses in a perovskite solar cell and discriminate between productive, that is, the fraction of light absorbed by the perovskite that generates photocurrent, and parasitic or nonproductive absorption in the other component layers. The solar spectrum weighted integrated absorptance (SSWIA), defined as the fraction of light absorbed by the perovskite weighted across the solar spectrum, between 400 and $800 \mathrm{~nm}$, is $82.9 \%$ for the cell under investigation. Note that the total fraction of unproductive light absorbed is below $6 \%$ throughout the spectrum, being ca. $2 \%$ at $500 \mathrm{~nm}$. In contrast, the specular reflectance is $>5 \%$ across the spectrum, reaching values as high as ca. $15 \%$ at 750 $\mathrm{nm}$.

The external quantum efficiency (EQE) measured from a representative perovskite PV device is displayed as a black solid line in Figure $3 \mathrm{c}$. EQE is defined as the ratio of collected carriers to the incident photons. The internal quantum efficiency (IQE) of a solar cell is typically calculated from the ratio of its $\mathrm{EQE}$ and $\mathrm{A}^{10}$ The presence of parasitic absorption in the device leads to underestimate the IQE of the solar cell. Using our optical model, it is possible to calculate only the fraction of light absorbed within the perovskite absorber (see Figure $3 \mathrm{~b}$ ), which enables an accurate determination of the IQE. The IQE, shown as an orange dashed line in Figure $3 c$, is relatively stable over ca. $80 \%$ from 450 to $750 \mathrm{~nm}$, indicating that, despite the fact that each wavelength is preferentially absorbed in a different region of the perovskite layer, as revealed by profiles like the one shown in Figure 3a, photogenerated carriers are collected with equal efficiency in the contacts. Our optical analysis shows that it should be possible to further improve the efficiency of perovskite solar cells by optimizing harvesting of solar radiation in the far red region of the electromagnetic spectrum. This can be achieved by integrating optical elements that selectively enhance the productive absorption of the cell at those wavelengths or by coating the outer glass with broadband antireflection coatings to minimize reflection losses.

Finally, in Figure 4a, we make use of our theoretical model to extrapolate the SSWIA of devices featuring infiltrated mesostructured $\mathrm{Al}_{2} \mathrm{O}_{3}$ scaffolds as a function of the thickness of the hole transport layer (HTL). Please note that the SSWIA hereby defined would be directly proportional to the estimated short circuit photocurrent if a charge collection efficiency of $100 \%$ is assumed. It can be observed that the SSWIA is larger for thicker scaffolds due to a larger amount of absorbing material. More interestingly, the SSWIA shows an oscillatory behavior with the spiro-OMeTAD layer thickness for a fixed scaffold. Similar oscillatory profiles are attained for scaffolds of different thickness, as shown in Figure 4a. As an example, we observe that for a $300 \mathrm{~nm}$ thick perovskite-infiltrated scaffold, the SSWIA is $72 \%$ for cells comprising an HTL with thickness of 100 or $320 \mathrm{~nm}$, whereas it reaches $75 \%$ for a $220 \mathrm{~nm}$ thick HTL. Figure $4 \mathrm{~b}$ shows the spectral dependence of the fraction of light absorbed by the perovskite material ( $\left.A_{\text {perovskite }}\right)$ when infiltrated in mesostructured scaffolds of the same thickness but different composition, namely, $\mathrm{Al}_{2} \mathrm{O}_{3}, \mathrm{SiO}_{2}$, and $\mathrm{TiO}_{2}$. The differences observed may be attributed to the difference in the refractive index of the scaffolds, where $\mathrm{TiO}_{2}$ is the highest and $\mathrm{SiO}_{2}$ is the lowest among the ones considered. One may note that the higher the refractive index, the higher the reflectance, and therefore the lower the absorptance. Such variations can be further quantified through the SSWIA parameter, attaining
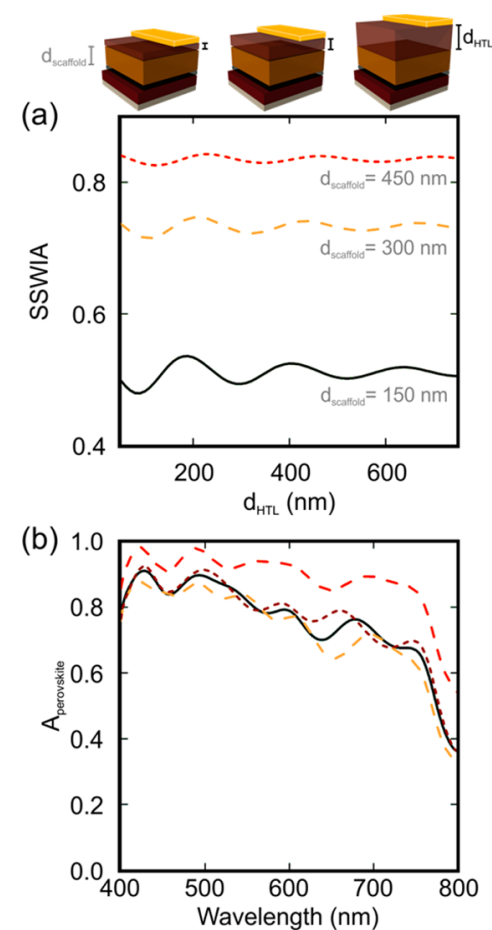

Figure 4. (a) Calculated solar spectrum weighted integrated absorptance (SSWIA) as a function of the hole transport layer (HTL) thickness for photovoltaic devices comprising perovskiteinfiltrated mesoporous $\mathrm{Al}_{2} \mathrm{O}_{3}$ scaffolds with a thickness of 150 (black solid line), 300 (orange dashed line), and $450 \mathrm{~nm}$ (red short dashed line). (b) Spectral dependence of the productive absorptance $\left(A_{\text {perovskite }}\right)$ of solar cells, in which the perovskite infiltrates a $300 \mathrm{~nm}$ thick mesostructured scaffold made of: $\mathrm{SiO}_{2}$ (brown short dashed line), $\mathrm{Al}_{2} \mathrm{O}_{3}$ (black solid line), and $\mathrm{TiO}_{2}$ (orange dashed line). For comparison, we present as red dashed line the $A_{\text {perovskite }}$ of a solar cell that integrates a solid perovskite film of the same thickness, that is, 300 $\mathrm{nm}$. We note that the perovskite absorber occupies only $50 \%$ of the volume within the photoactive region and that there is no capping layer considered in the calculations.

values of 72,75 , and $76 \%$ for the perovskite-infiltrated $\mathrm{TiO}_{2}$, $\mathrm{Al}_{2} \mathrm{O}_{3}$, and $\mathrm{SiO}_{2}$ scaffolds, respectively. In Figure $4 \mathrm{~b}$, we also plot the $A_{\text {perovsite }}$ for a solar cell that integrates a solid perovskite film of the same thickness $(300 \mathrm{~nm})$. The presence of a larger amount of perovskite absorber within the photoactive region leads to almost full absorption, reaching a SSWIA value as high as $88 \%$. Our model also serves to calculate the thickness of the scaffold that would provide a similar SSWIA, attaining thickness values of 600,570 , and $540 \mathrm{~nm}$ for mesostructured scaffolds made of $\mathrm{TiO}_{2}, \mathrm{Al}_{2} \mathrm{O}_{3}$, and $\mathrm{SiO}_{2}$, respectively. Thus, our analysis presents the possibility of finding the material features needed to attain scaffold based perovskite PV devices in which light harvesting is as efficient as in flat bulk perovskite layers. It can also be helpful for optimizing vapor-deposited cells in which extremely uniform films with an accurate thickness control can be achieved. ${ }^{11}$

In summary, we have developed a rigorous theoretical model that describes the optical behavior of organic-inorganic halide perovskite solar cells. Our theoretical results were confirmed with experimental measurements from state-of-the-art solutionprocessed devices, although the proposed model could be also employed to describe the optical response of other layered perovskite-based PV devices, for instance, planar heterojunction solar cells prepared by evaporation solution processing. It 
serves to visualize the spatial distribution of the radiation within the cell, which allows quantifying the fraction of light absorbed by each constituent of the device, where it is possible to discern the radiation that actually contributes to generate photocurrent. This allows us to perform an accurate estimation of the internal quantum efficiency of the solar cell. Finally, we have extrapolated our model to describe the influence, due to optical interference, of the features of the different constituents of the cell, such as the thickness of the hole transporting layer, on the light harvesting of solar radiation by the PV device. Insight is also provided into the effect of scaffolds of different composition on the total amount of light harvested by the perovskite absorber as well as on the thickness required for each type of scaffold to reach the same light absorption than a flat dense perovskite layer. Our results hence open a new avenue for the optical design of perovskite solar cells with improved efficiency.

\section{EXPERIMENTAL METHODS}

Synthesis of Perovskite. Methylamine iodide $\left(\mathrm{CH}_{3} \mathrm{NH}_{3} \mathrm{I}\right)$ was prepared by reacting methylamine, $33 \mathrm{wt} \%$ in ethanol (SigmaAldrich), with hydroiodic acid (HI) 57 wt \% in water (SigmaAldrich), at room temperature. Typical quantities employed are $24 \mathrm{~mL}$ of methylamine, $10 \mathrm{~mL}$ of $\mathrm{HI}$, and $100 \mathrm{~mL}$ of ethanol. Upon drying at $100{ }^{\circ} \mathrm{C}$, a white powder was formed, which was placed overnight in a vacuum oven and rinsed with ethanol before use. 9 To obtain the perovskite solution, we dissolved both the $\mathrm{CH}_{3} \mathrm{NH}_{3} \mathrm{I}$ and the $\mathrm{PbCl}_{2}$ (Sigma-Aldrich) in anhydrous $N, N$-dimethylformamide (DMF) at a 3:1 molar ratio with final concentrations of ca. 40 wt \%.

A modification of this method, in which $\mathrm{Pb}\left(\mathrm{CH}_{3} \mathrm{COO}\right)_{2}$ is used as perovskite precursor instead of $\mathrm{PbCl}_{2}$, was employed to fabricate smooth perovskite flat layers with improved film morphology while keeping the chemical composition unchanged.

Device Fabrication. All details regarding the fabrication of the solar cells can be found elsewhere. ${ }^{34}$ In short, FTO patterned glass (Pilkington TEC $7,7 \Omega /$ sq) was cleaned sequentially in Hellmanex, Milli-Q water, acetone, ethanol, and oxygen plasma. A $\mathrm{TiO}_{2}$ compact layer was deposited by spin coating a $253 \mathrm{mM}$ titanium isopropoxide precursor (diluted in a mildly acidic anhydrous ethanol solution) at a speed of $2000 \mathrm{rpm}$ for $60 \mathrm{~s}$, followed by sintering at $500{ }^{\circ} \mathrm{C}$ during $45 \mathrm{~min}$. After cooling to room temperature, an $\mathrm{Al}_{2} \mathrm{O}_{3}$ scaffold was spin coated at 2500 $\mathrm{rpm}$ for $45 \mathrm{~s}$ from a colloidal dispersion of $<50 \mathrm{~nm} \mathrm{Al}_{2} \mathrm{O}_{3}$ nanoparticles (Sigma-Aldrich, product number 702129), followed by drying at $150{ }^{\circ} \mathrm{C}$ for $30 \mathrm{~min}$. Throughout all experiments the scaffold thickness was kept constant at ca. 350 $\mathrm{nm}$ by diluting the $\mathrm{Al}_{2} \mathrm{O}_{3}$ nanoparticles dispersion with ethanol in a 1:2 volume ratio. To fabricate the solar cells, we infiltrated $\mathrm{Al}_{2} \mathrm{O}_{3}$ scaffolds using a 40 wt $\%$ perovskite solution by spincoating at $2000 \mathrm{rpm}$ for $45 \mathrm{~s}$ in a nitrogen-filled glovebox. After the spin-coating process, samples were annealed at $100{ }^{\circ} \mathrm{C}$ for 2 h. The spiro-OMeTAD hole-transporting layer was then deposited from a $80 \mathrm{mM}$ chlorobenzene solution containing additives of lithium bis(trifluoromethanesulfonyl)imide and 4tert-butylpyridine. Finally, gold electrodes were thermally evaporated under vacuum of ca. $10^{-6}$ Torr, at a rate of ca. $0.1 \mathrm{~nm} / \mathrm{s}$, to complete the devices.

Structural Characterization. Cross-section field-emission SEM images of PV devices were taken by using a microscope Hitachi 5200 operating at $5 \mathrm{kV}$.
Optical Characterization. The spectral dependence of the reflectance and transmittance of the solar cells was obtained using an integrating sphere (Labsphere RTC-060-SF), a halogen lamp (Ocean Optics HL-2000) as light source, and a spectrometer (Ocean Optics USB 2000) as detector.

Photovoltaic Characterization. The current density-voltage curves were measured (2400 Series SourceMeter, Keithley Instruments) under simulated AM 1.5 sunlight at $100 \mathrm{~mW}$ $\mathrm{cm}^{-2}$ irradiance generated by an Abet Class $\mathrm{AAB}$ sun 2000 simulator, with the intensity calibrated with an NREL calibrated KG5 filtered $\mathrm{Si}$ reference cell. The mismatch factor was calculated to be $<1 \%$. The solar cells were masked with a metal aperture to define the active area, typically $0.0625 \mathrm{~cm}^{2}$ (measured individually for each mask), and measured in a light-tight sample holder to minimize any edge effects and ensure that the reference and test cells are located in the same spot under the solar simulator during measurement. EQE was measured via Fourier transform photocurrent spectroscopy using a Bruker Vertex 80v Fourier transform interferometer with tungsten-halogen lamp source, $\mathrm{CaF}_{2}$ beam splitter, a Stanford Research SR570 current preamplifier, and custombuilt control electronics. A forward bias of $1.4 \mathrm{~V}$ was applied to devices for a period of $20 \mathrm{~s}$ before photocurrent spectra were recorded. EQE spectra were calibrated to a Newport-calibrated reference Si solar cell with a known EQE. The solar cells were masked with a metal aperture to define an active area of 0.0625 $\mathrm{cm}^{2}$.

\section{ASSOCIATED CONTENT}

\section{S Supporting Information}

Additional photovoltaic and optical characterization is included. This material is available free of charge via the Internet at http://pubs.acs.org.

\section{AUTHOR INFORMATION}

\section{Corresponding Author}

*E-mail: h.miguez@csic.es.

\section{Notes}

The authors declare no competing financial interest.

During the review process of this manuscript two related articles discussing the optical modeling of planar perovskite solar cells were published (refs 35 and 36).

\section{ACKNOWLEDGMENTS}

The research leading to these results has received funding from the European Research Council under the European Union's Seventh Framework Programme (FP7/2007-2013)/ERC grant agreement no. 307081 (POLIGHT) and HYPER Project, the Spanish Ministry of Economy and Competitiveness under grant MAT2011-23593 and the EPSRC Supergen. M.A. is grateful to "La Caixa" Foundation for its financial support. FESEM characterization was performed at CITIUS, and we are grateful for its support.

\section{REFERENCES}

(1) Park, N.-G. Organometal Perovskite Light Absorbers toward a 20\% Efficiency Low-Cost Solid-State Mesoscopic Solar Cell. J. Phys. Chem. Lett. 2013, 4, 2423-2429.

(2) Bisquert, J. The Swift Surge of Perovskite Photovoltaics. J. Phys. Chem. Lett. 2013, 4, 2597-2598.

(3) Snaith, H. J. Perovskites: The Emergence of a New Era for LowCost, High-Efficiency Solar Cells. J. Phys. Chem. Lett. 2013, 4, 36233630. 
(4) Gao, P.; Grätzel, M.; Nazeeruddin, M. K. Organohalide Lead Perovskites for Photovoltaic Applications. Energy Environ. Sci. 2014, 7, $2448-2463$.

(5) Kim, H.-S.; Im, S. H.; Park, N.-G. Organolead Halide Perovskite: New Horizons in Solar Cell Research. J. Phys. Chem. C 2014, 118, $5615-5625$.

(6) Research Cell Efficiency Records. NREL (http://www.nrel.gov/ ncpv/).

(7) Kojima, A.; Teshima, K.; Shirai, Y.; Miyasaka, T. Organometal Halide Perovskites As Visible-Light Sensitizers for Photovoltaic Cells. J. Am. Chem. Soc. 2009, 131, 6050-6051.

(8) Kim, H.-S; Lee, C.-R.; Im, J.-H; Lee, K.-B.; Moehl, T.; Marchioro, A.; Moon, S.-J.; Humphry-Baker, R.; Yum, J. H.; Moser, J. E.; et al. Lead Iodide Perovskite Sensitized All-Solid-State Submicron Thin Film Mesoscopic Solar Cell with Efficiency Exceeding 9\%. Sci. Rep. 2012, 2, 591.

(9) Lee, M. M.; Teuscher, J.; Miyasaka, T.; Murakami, T. N.; Snaith, H. J. Efficient Hybrid Solar Cells Based on Meso-Superstructured Organometal Halide Perovskites. Science 2012, 338, 643-647.

(10) Burschka, J.; Pellet, N.; Moon, S.-J.; Humphry-Baker, R.; Gao, P.; Nazeeruddin, M. K.; Grätzel, M. Sequential Deposition As a Route to High-Performance Perovskite-Sensitized Solar Cells. Nature 2013, 499, 316-319.

(11) Liu, M.; Johnston, M. B.; Snaith, H. J. Efficient Planar Heterojunction Perovskite Solar Cells by Vapour Deposition. Nature 2013, 501, 395-398.

(12) Heo, J. H.; Im, S. H.; Noh, J. H.; Mandal, T. N.; Lim, C.-S.; Chang, J. A.; Lee, Y. H.; Kim, H.; Sarkar, A.; Nazeeruddin, M. K.; et al. Efficient Inorganic-Organic Hybrid Heterojunction Solar Cells Containing Perovskite Compound and Polymeric Hole Conductors. Nat. Photonics 2013, 7, 486-491.

(13) Liu, D.; Kelly, T. L. Perovskite Solar Cells with a Planar Heterojunction Structure Prepared Using Room-Temperature Solution Processing Techniques. Nat. Photonics 2014, 8, 133-138.

(14) Mei, A.; Li, X.; Liu, L.; Ku, Z.; Liu, T.; Rong, Y.; Xu, M.; Hu, M.; Chen, J.; Yang, Y.; et al. A Hole-Conductor-Free, Fully Printable Mesoscopic Perovskite Solar Cell with High Stability. Science 2014, 345, 295-298.

(15) Zhou, H.; Chen, Q.; Li, G.; Luo, S.; Song, T.; Duan, H.-S.; Hong, Z.; You, J.; Liu, Y.; Yang, Y. Interface Engineering of Highly Efficient Perovskite Solar Cells. Science 2014, 345, 542-546.

(16) Jeon, N. J.; Noh, J. H.; Kim, Y. C.; Yang, W. S.; Ryu, S.; Seok, S. Solvent Engineering for High-Performance Inorganic-organic Hybrid Perovskite Solar Cells. Nat. Mater. 2014, 13, 897-903.

(17) Son, D.-Y.; Im, J.-H.; Kim, H.-S.; Park, N.-G. 11\% Efficient Perovskite Solar Cell Based on ZnO Nanorods: An Effective Charge Collection System. J. Phys. Chem. C 2014, 118, 16567-16573.

(18) Hu, Q.; Wu, J.; Jiang, C.; Liu, T.; Que, X.; Zhu, R.; Gong, Q. Engineering of Electron-Selective Contact for Perovskite Solar Cells with Efficiency Exceeding 15\%. ACS Nano 2014, 10, 10161-10167.

(19) Im, J.-H.; Jang, I.-H.; Pellet, N.; Grätzel, M.; Park, N.-G. Growth of $\mathrm{CH}_{3} \mathrm{NH}_{3} \mathrm{PbI}_{3}$ Cuboids with Controlled Size for High-Efficiency Perovskite Solar Cells. Nat. Nanotechnol. 2014, 9, 927-932.

(20) Polman, A.; Atwater, H. A. Photonic Design Principles for Ultrahigh-Efficiency Photovoltaics. Nat. Mater. 2012, 11, 174-177.

(21) De Wolf, S.; Holovsky, J.; Moon, S.-J.; Löper, P.; Niesen, B.; Ledinsky, M.; Haug, F.-J.; Yum, J.-H.; Ballif, C. Organolead Halide Perovskite: Sharp Optical Absorption Edge and Its Relation to Photovoltaic Performance. J. Phys. Chem. Lett. 2014, 5, 1035-1039.

(22) Sadhanala, A.; Deschler, F.; Thomas, T. H.; Dutton, S. E.; Goedel, K. C.; Hanusch, F. C.; Lai, M. L.; Steiner, U.; Bein, T.; Docampo, P.; et al. Preparation of Single-Phase Films of $\mathrm{CH}_{3} \mathrm{NH}_{3} \mathrm{~Pb}-$ $\left(\mathrm{I}_{1-\mathrm{x}} \mathrm{Br}_{\mathrm{x}}\right)_{3}$ with Sharp Optical Band Edges. J. Phys. Chem. Lett. 2014, 5, 2501-2505.

(23) Manser, J. S.; Kamat, P. V. Band Filling with Free Charge Carriers in Organometal Halide Perovskites. Nat. Photonics 2014, 8, 737-743.
(24) Zhu, X.; Su, H.; Marcus, R. A.; Michel-Beyerle, M. E. Computed and Experimental Absorption Spectra of the Perovskite $\mathrm{CH}_{3} \mathrm{NH}_{3} \mathrm{PbI}_{3}$. J. Phys. Chem. Lett. 2014, 5, 3061-3065.

(25) Chen, C.-W.; Hsiao, S.-Y.; Chen, C.-Y.; Kang, H.-W.; Huang, Z.Y.; Lin, H.-W. Optical Properties of Organometal Halide Perovskite Thin Films and General Device Structure Design Rules for Perovskite Single and Tandem Solar Cells. J. Mater. Chem. A 2014, DOI: $10.1039 / \mathrm{C} 4 \mathrm{TA}$ 05237D.

(26) Yeh, P. Optical Waves in Layered Media; John Wiley \& Sons: Hoboken, NJ, 2005.

(27) Wenger, S.; Schmid, M.; Rothenberger, G.; Gentsch, A.; Grätzel, M.; Schumacher, J. O. Coupled Optical and Electronic Modeling of Dye-Sensitized Solar Cells for Steady-State Parameter Extraction. J. Phys. Chem. C 2011, 115, 10218-10229.

(28) Moule, A. J.; Snaith, H. J.; Kaiser, M.; Klesper, H.; Huang, D. M.; Grätzel, M.; Meerholz, K. Optical Description of Solid-State DyeSensitized Solar Cells. I. Measurement of Layer Optical Properties. J. Appl. Phys. 2009, 106, 073111.

(29) Palik, E. D. Handbook of Optical Constants of Solids; Academic Press: San Diego, CA, 1985.

(30) Xing, G.; Mathews, N.; Lim, S.-S.; Yantara, N.; Liu, X.; Dharani, S.; Grätzel, M.; Mhaisalkar, S.; Sun, T. C. Low-Temperature SolutionProcessed Wavelength-Tunable Perovskites for Lasing. Nat. Mater. 2014, 13, 476-480.

(31) Malitson, I. H. Refraction and Dispersion of Synthetic Sapphire. J. Opt. Soc. Am. 1962, 52, 1377-1379.

(32) Lozano, G.; Colodrero, S.; Caulier, O.; Calvo, M. E.; Míguez, H. Theoretical Analysis of the Performance of One-Dimensional Photonic Crystal-Based Dye-Sensitized Solar Cells. J. Phys. Chem. C 2010, 114, 3681-3687.

(33) Santbergen, R.; Smets, A. H. M.; Zeman, M. Optical Model for Multilayer Structures with Coherent, Partially Coherent and Incoherent Layers. Opt. Express 2013, 21, A262-A267.

(34) Ball, J. M.; Lee, M. M.; Hey, A.; Snaith, H. J. Low-Temperature Processed Meso-Superstructured to Thin-Film Perovskite Solar Cells. Energy Environ. Sci. 2013, 6, 1739-1743.

(35) Lin, Q.; Armin, A.; Nagiri, R. C. R.; Burn, P. L.; Meredith, P. Electro-Optics of Perovskite Solar Cells. Nat. Photonics 2014, DOI: 10.1038/nphoton.2014.284.

(36) Ball, J. M.; Stranks, S. D.; Hörantner, M. T.; Hüttner, S.; Zhang, W.; Crossland, E. J. W.; Ramirez, I.; Moritz, R.; Johnston, M. B.; Friend, R. H.; et al. Optical Properties and Limiting Photocurrent of Thin-Film Perovskite Solar Cells. Energy Environ. Sci. 2014, DOI: $10.1039 / C 4 E E 03224 A$. 